\title{
Genetically Enhanced Performance of a UTRA-like Time-Division Duplex CDMA Network
}

\author{
Song Ni, Lajos Hanzo ${ }^{1}$ \\ School of Electronics and Computer Science, Univ. of Southampton, SO17 1BJ, UK. \\ Tel: +44-(0)23-8059-3125, Fax: +44-(0)23-8059-4508 \\ Email:1h1@ecs.soton.ac.uk http://www-mobile.ecs.soton.ac.uk
}

\begin{abstract}
In this contribution a Dynamic Channel Allocation (DCA) algorithm is developed, which minimizes the amount of Multi-User Interference (MUI) experienced at the Base Stations (BSs) by employing Genetic Algorithms (GAs). A GA is utilized for finding a suboptimum, but highly beneficial Uplink (UL) or Downlink (DL) Timeslot (TS) allocation for improving the achievable performance of the third generation UTRA system's Time Division Duplex (TDD) mode. It is demonstrated that a GA-assisted UL/DL timeslot scheduling scheme may avoid the severe BS to BS inter-cell interference potentially inflicted by the UTRA TDD CDMA air interface owing to allowing all TSs to be used both in the UL and DL
\end{abstract}

\section{INTRODUCTION}

The UTRA Time Division Duplex (TDD) mode was contrived for the sake of improving the achievable network performance by assigning all the timeslots on a demand basis to the uplink and downlink. However, we demonstrated in [1] that the arbitrary allocation of uplink and downlink timeslots may result in an excessive BS-to-BS interference and hence in a potentially reduced number of system users. We therefore invoked both adaptive modulation and adaptive beamforming for the sake of mitigating this TDD-specific problem and demonstrated that with their advent the number of users supported may become similar, but still somewhat inferior in comparison to that of an Frequency Division Duplex (FDD) system. In this contribution our research evolves further and as a design alternative, we will apply a $\mathrm{Ge}-$ netic Algorithm (GA) [2] for improving the achievable performance of the UTRA TDD mode. More specifically, in [1] we demonstrated that the employment of adaptive arrays in conjunction with Adaptive Quadrature Amplitude Modulation (AQAM) [3] limited the detrimental effects of co-channel interference on the UTRA-like TDD/CDMA system and resulted in performance improvements both in terms of the achievable call quality and the number of users supported. However, in comparison to a UTRA-like FDD/CDMA system, the capacity of the UTRA-like TDD/CDMA cellular system was shown to remain somewhat poorer under the same propagation conditions. It was shown that the TDD mode is more prone to avalanche-like teletraffic overload and its carried teletraffic is up to a factor two lower than that of the FDD mode. Again, this is because in the TDD mode Mobile Stations (MSs) can interfere both with Base Stations (BSs) as well as with each other. The same holds for BSs, which can interfere with

The financial support of the EPSRC, UK and the EU under the auspices of the Phonix, Newcom and Nexway projects is gratefully acknowledged. both MSs and other BSs owing to potentially using all timeslots in both the uplink and downlink. The resultant additional interference has a detrimental impact on the system's capacity. These conclusions were also corroborated by $\mathrm{Wu}$ [4], who pointed out that the inter-cell BS-to-BS interference substantially decreases the system's user capacity. Hence we can increase the total system capacity by reducing the BS-to-BS interference. One way of achieving a reduced BS-to-BS interference is by invoking beamforming [5], since the BS becomes capable of focusing its transmitted signal energy on the served MSs, while creating a radiation null in the direction of the adjacent BSs, as we have also shown in [1]. However, this desirable goal may only be achieved, if there are no MSs roaming in the immediate vicinity of the line between the serving BS and the neighbouring BS. Hence the achievable capacity of the beamforing aided UTRA-like TDD/CDMA system remains limited.

In order to mitigate these performance limitations, we designed a GA-assisted TS-scheduling scheme for the UTRA-like TDD/CDMA system. A Dynamic Channel Allocation (DCA) algorithm was developed, which minimizes the amount of MUI experienced at the BSs by employing GAs $[6,7]$.

The structure of this paper is as follows. In Section 2 we will first describe our GA-aided UTRA-like TDD/CDMA system model used. The numerical results characterizing the various interference scenarios in terms of the number of users supported by the GA-assisted TDD/CDMA system are compared to those of the TDD/CDMA system dispensing with GAs in Section 3. Finally, we conclude our discussions in Section 4. Let us now commence our discourse by briefly highlighting how GAs may be used for enhancing the UTRA/TDD system's performance.

\section{THE GENETICALLY ENHANCED UTRA-LIKE TDD/CDMA SYSTEM}

Recently substantial advances have been made in the context of diverse GA-aided wireless receivers, such as CDMA multiuser detectors [2], beamforming [8] and Space Division Multiple Access (SDMA) aided OFDM [9]. Genetic algorithms have been used as robust guided stochastic search algorithms for solving various optimization problems, as diverse as multiprocessor scheduling [10], topology design and bandwidth allocation in ATM networks [11], for improving the performance of channel allocation in cellular networks [12], for code design [13] and code set selection in optical CDMA networks [14]. Despite establishing GAs as a useful optimization tool in numerous applications, the employment of GAs in the network layer of mobile communications, has been extremely rare. In order to probe further, in this contribution genetic algorithms have been utilized by a UTRA-like TDD/CDMA system, where the GA-assisted timeslot al- 


\begin{tabular}{|c|c|c|c|c|c|c|c|c|c|c|c|c|}
\hline$f_{i j}$ & & & & & & slot In & & & & & & \\
\hline & 1 & 2 & 3 & 4 & 5 & $\ldots$ & & & & & & $m$ \\
\hline 1 & 0 & 1 & 0 & 0 & 1 & $\ldots$ & 0 & 0 & 0 & 0 & 0 & 1 \\
\hline 2 & 0 & 0 & 0 & 0 & 0 & $\ldots$ & 0 & 1 & 0 & 0 & 0 & 0 \\
\hline 3 & 0 & 0 & 1 & 0 & 0 & $\ldots$ & 0 & 0 & 0 & 1 & 0 & 0 \\
\hline 11 Index & & & & & & & & & & & & \\
\hline & 1 & 0 & 1 & 0 & 0 & $\ldots$ & 0 & 0 & 1 & 0 & 1 & 0 \\
\hline & 0 & 0 & 0 & 1 & 0 & $\ldots$ & 1 & 0 & 0 & 0 & 0 & 0 \\
\hline$n$ & 1 & 0 & 0 & 0 & 0 & $\ldots$ & 0 & 0 & 0 & 1 & 0 & 0 \\
\hline
\end{tabular}

Figure 1: Timeslot allocation matrix used by the GA

locator assigns either uplink or downlink timeslots to MSs or BSs, while maintaining certain Quality of Service (QoS) guarantees.

The aim of this design is to maximize the achievable UTRA-like TDD/CDMA network's capacity, quantified in terms of the mean normalized carried traffic expressed in units of Erlang $/ \mathrm{km}^{2} / \mathrm{MHz}$. The performance metrics used to quantify the quality of service have been described in [1]. Recall that the false termination (FT) probability, which is also often referred to as the call dropping probability, $P_{F T}$, quantifies the probability that a call is forced to be prematurely terminated. This may be the consequence of an insufficiently high SINR encountered during the call, which is not remedied by an intra-cell handover, either due to the lack of available channels, or owing to an insufficient improvement of the SINR, which leads to successive outages and eventually to a dropped call. Calls may also suffer from forced termination, when a mobile enters a heavily loaded cell, which either suffers from a poor average SINR or has no available channels for the mobile to handover to. The main performance limiting factors are constituted by potentially high interference levels and low maximum affordable transmit powers, often resulting in excessive call dropping rates. Since a dropped call constitutes an annoyance from a user's viewpoint, the users' SINR value has to be maintained safely, but not excessively above the target SINR value.

The interference experienced at the mobile can be divided into interference due to the signals transmitted to other mobiles from the same base station, which is known as intra-cell interference, and that encountered owing to the signals transmitted to other mobiles from other basestations, as well as to other basestations from other mobiles, which is termed inter-cell interference.

The uplink differs from the downlink in that the multiple access interference is typically asynchronous in the uplink due to the uncoordinated transmissions of the mobile stations, roaming at different distances for the BS whereas it may remain synchronous in the downlink since all signals are transmitted by the BS. To elaborate a little further, all the synchronous downlink signals of the users sharing a given timeslot are assumed to arrive at the MS via the same propagation channel. The pathloss and shadow fading are updated on a $10 \mathrm{~ms}$ UTRA frame by frame basis, once every 15 timeslots [2]. If this channel is dispersive, it does affect the orthogonality of each of the OVSF codes, but the amount of interference inflicted remains lower than in case of the asynchronous uplink, where all multipath components of the asynchronous users arrive at different times at the $\mathrm{BS}$, as discussed in [15]. A possible solution for mitigating the problem of OVSF code orthogonality degradation imposed by channeldispersion-induced linear distortions is employing Multi-User Detectors (MUDs) [2] at the base stations.

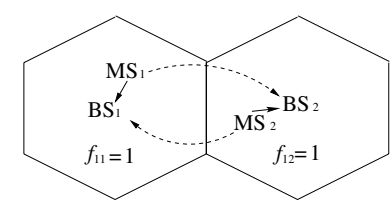

( a )

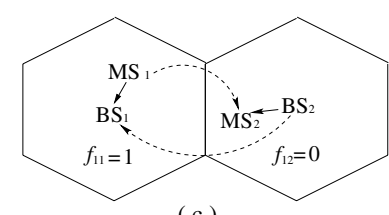

(c)

$\begin{array}{ll}\text { Timeslot ID: } & 1 \\ \text { Number of BSs: } & 2\end{array}$ $\begin{array}{ll}\text { Number of BSs: } & 2 \\ \text { Number of MSs: } & 2\end{array}$

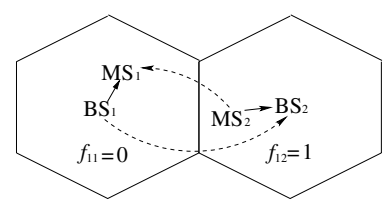

( b )

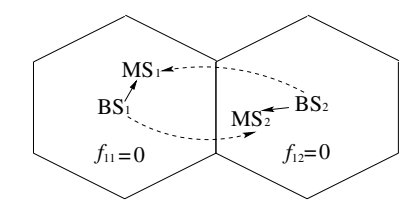

(d)

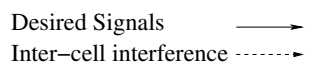

Figure 2: An example of UL/DL timeslot allocation options.

Following the above introductory considerations, let us represent the GA's solution space $F$ as an $n \times m$-dimensional binary matrix, where $n$ is the number of radio cells and $m$ is the total number of timeslots. Explicitly, the total number of timeslots is the product of the number of traffic cells, the number of RF carriers per cell and the number of timeslots per carrier. Each element $f_{i j}$ in the matrix is either a binary one or zero, as shown in Figure 1, where a one corresponds to UL allocation and vice versa. The optimisation task to be solved by the GA is to find the best UL/DL constellation of all the TSs of all the cells for all UTRA TDD frames. Naturally, this optimisation task would impose an excessive complexity and hence a computationally efficient suboptimum solution is sought.

In our previous investigations [1] we quantified the achievable performance of the UTRA TDD/CDMA system, demonstrating that significant performance improvements can be achieved as a direct result of the interference rejection capabilities of the adaptive antenna arrays and adaptive modulation invoked. Hence the reduction of the interference improved the system's performance. The amount of inter-cell interference imposed depends on the angle of arrival of the interference imposed by the adjacent radio cell. Again, if the timeslot in the interfering cell is used as an uplink timeslot, then we have $f_{i j}=1$ in Table 1 , and vice versa. A simple example of the possible timeslot allocation scenarios is given in Figure 2, portraying four possible timeslot allocation scenarios for two BSs and two MSs.

More specifically, in the scenario of Figure 2 (a) and (c), $B S_{1}$ experiences two types of inter-cell interference, namely $M S_{2} \rightarrow B S_{1}$ and $B S_{2} \rightarrow B S_{1}$, respectively. Similarly, in the scenario seen in Figure 2 (b) and (d), $M S_{1}$ also experiences two different types of intercell interferences imposed by the neighbouring cell, which manifests itself as $M S_{2} \rightarrow M S_{1}$ and $B S_{2} \rightarrow M S_{1}$ interference, respectively. In [4] it was shown that the major source of interference is constituted by the BS-to-BS interference, as a consequence of the BS's high signal power and the near-LOS propagation conditions prevailing between the high-elevation BSs. Hence we can avoid encountering a high $B S \rightarrow B S$ inter-cell interference by appropriately scheduling the allocation of timeslots. Interference is inherent in cellular systems, and it is a challenging task to control it in practice due to the presence of random propagation effects. If there are $n$ potential serving BSs in an area, for each timeslot there are $2^{n}$ ways of allocating it to a specific BS either in the uplink or downlink. As argued before, an optimal timeslot allocation algorithm would have to tentatively invoke all $2^{n}$ 


\begin{tabular}{|l|r|l|r|}
\hline Parameter & Value & Parameter & Value \\
\hline Frame length & $10 \mathrm{~ms}$ & Timeslots per frame & 15 \\
Target $E_{b} / N_{o}$ & $8.0 \mathrm{~dB}$ & Outage $E_{b} / N_{o}$ & $6.6 \mathrm{~dB}$ \\
Low Quality (LQ) Outage $E_{b} / N_{o}$ & $7.0 \mathrm{~dB}$ & BS Pilot Power & $-5 \mathrm{dBm}$ \\
BS/MS Minimum TX Power & $-44 \mathrm{dBm}$ & BS/MS Maximum TX Power & $+21 \mathrm{dBm}$ \\
Pathloss exponent & -3.5 & Noisefloor & $-100 \mathrm{dBm}$ \\
Power control SINR hysteresis & $1 \mathrm{~dB}$ & Cell radius & $150 \mathrm{~m}$ \\
Spreading factor & 16 & Modulation scheme & $4 \mathrm{QAM}$ \\
Max new-call queue-time & $5 \mathrm{~s}$ & Average inter-call time & $300 \mathrm{~s}$ \\
Average call length & $60 \mathrm{~s}$ & User speed & $1.34 \mathrm{~m} / \mathrm{s}$ \\
\hline
\end{tabular}

Table 1: Simulation parameters of the UTRA-like TDD/CDMA cellular network.

possible TS allocations, in order to find the best one, when a new TS has to be allocated to a user, who is initiating a new call. However, since this new TS allocation affects the entire system's interference patterns, the complexity of the optimum full-search algorithm would become excessive. In order to reduce the complexity of the associated decisions, we invoked a genetic algorithm for determining the advantageous scheduling of uplink and downlink timeslots. The genetic algorithm uses an objective function [2] to determine how 'fit' each UL/DL TS allocation is for survival in the consecutive generations of the GA, when ranking potential UL/DL TS allocation configurations. For instance, the aim of the GA is to determine in our example provided in Figure 2 of this section, which particular UL/DL TS allocation of the total of four different options has the best overall connection quality, lower UL/DL average power consumption and lower interference level. The GA's objective function will be evaluated for a small fraction of the entire set of possible TS allocations, while aiming for a near-optimum solution.

\section{SIMULATION RESULTS}

In our initial investigations we do not impose any user requirements concerning the number of uplink and dowlink TSs requested, we simply aim for determining the best possible UL/DL system configuration, which would allow us to estimate the capacity of the system. The associated UTRA/TDD system parameters are described in Table 1. These investigations were conducted using a spreading factor of 16 . Given that the chip rate of UTRA is $3.84 \mathrm{Mchips} / \mathrm{sec}$, this spreading factor corresponds to a channel data rate of $3.84 \times 10^{6} / 16=240$ kbps. Applying 1/2 rate error correction coding would result in an effective data throughput of $120 \mathrm{kbps}$. A cell radius of $150 \mathrm{~m}$ was assumed and a pedestrian walking velocity of $3 \mathrm{mph}$ was used. The simulation area was constituted of 49 traffic cells using the infinite mesh of wrapped-around cellular structure of Figure 4.18 in [5] .

It was shown for example in Chapter 9 of [2] that the GA's performance is dependent on numerous factors, such as the population size $P$, the number of generations $Y$, the choice of the parents' selection method, as well as on a number of other genetic operations employed. In this section, we will quantify the system's achievable performance with the advent of GAs, and attempt to find an appropriate GA set-up and parameter configuration that are best suited for our optimization problem. The GA's parameters are summarized in Table 2. Our performance metrics are, as before, the call dropping or forced termination probability $P_{F T}$, the probability of low quality access $P_{\text {low }}$ and the mean transmission power [5]. The complexity of the GA is governed by the number of generations $Y$ required, in order to achieve a meritorious TS allocation. For the sake of simplicity, the computational complexity of the GA is quantified here in the con-

\begin{tabular}{|l|r|}
\hline Set-up/Parameter & Method/Value \\
\hline \hline $\begin{array}{l}\text { Individual initialization } \\
\text { method }\end{array}$ & Uniform Random \\
\hline Selection method & Fitness-Proportionate \\
\hline Cross-over operation & Single point \\
\hline Mutation operation & Uniform random bit flip \\
\hline Population size & Variable $P=4,10,20$ \\
\hline Generation size & Variable $Y=25,10,5$ \\
\hline Probability of Mutation & 0.1 \\
\hline Probability of Crossover & 0.9 \\
\hline Computational complexity & 100 \\
\hline
\end{tabular}

Table 2: Configuration of the GA used.

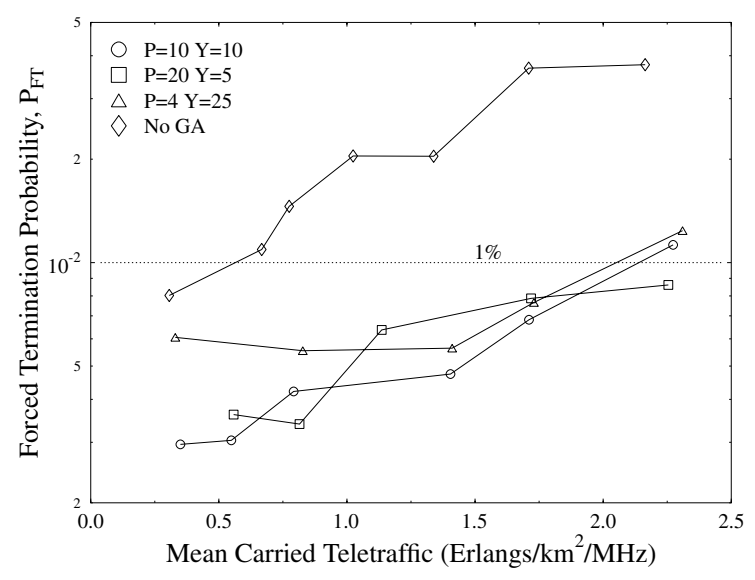

Figure 3: Call dropping probability versus mean carried traffic of the UTRA-like TDD/CDMA based cellular network both with and without GA-assisted timeslots allocation as well as with shadowing having a standard deviation of $3 \mathbf{d B}$ for $S F=16$.

text of the total number of objective function [2] evaluations, given by $P \times Y$.

Figure 3 shows the forced termination probability associated with a variety of traffic loads quantified in terms of the mean normalized carried traffic expressed in Erlangs $/ \mathrm{km}^{2} / \mathrm{MHz}$, when subjected to 0.5 $\mathrm{Hz}$ frequency shadowing having a standard deviation of $3 \mathrm{~dB}$. As observed in the figure, nearly an order of magnitude forced termination probability reduction has been achieved by employing GA-assisted timeslot scheduling compared to the "No GA" scheme refraining from using UL/DL TS optimization. In the context of the "No GA" scheme, the allocation of the uplink and downlink timeslots for each BS was 


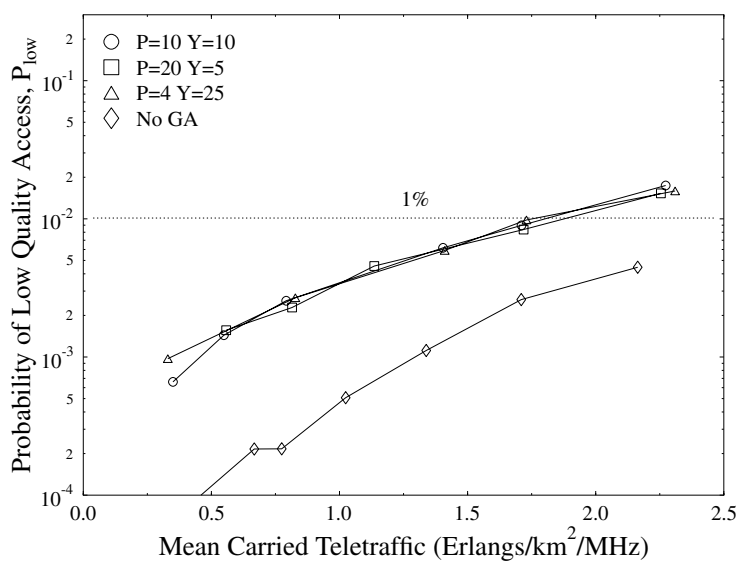

Figure 4: Probability of low quality access versus mean carried traffic of the UTRA-like TDD/CDMA based cellular network both with and without GA-assisted UL/DL TS-allocation as well as with shadowing having a standard deviation of $3 \mathrm{~dB}$ for $S F=16$.

fixed to a ratio of $7: 8$ (UL : DL). This fixed timeslot allocation may inflict a high $\mathrm{BS} \rightarrow \mathrm{BS}$ interference, when the serving cell is using uplink timeslots and the interfering cell is using downlink timeslots, as portrayed in Figure 2 (b) or (c). The associated high inter-cell interference may result in a poor SINR, which fails to satisfy the system's target SINR required for maintaining a high-quality connection and hence increases the probability of forced termination. By contrast, in the GA-assisted UTRA TDD/CDMA system each timeslot in a frame can be allocated to either the uplink or downlink, depending on the associated slot-SINR, potentially allowing us to allocate the timeslot by minimizing the inter-cell interference inflicted. As we mentioned in the previous section, for a UTRA/TDD system having $n \mathrm{BSs}$, there are $2^{n}$ possible UL/DL TS allocation schemes for each timeslot. In our simulated scenario there are 49 wrapped-around traffic cells and we use the infinite mesh of cellular structure portrayed in Figure 4.18 of [5], which results in the same amount of co-channel intereference for all cells. More explicitly, a 'desert island-like' 49-cell structure dispensing with the wrap-around of cells would result in an irrealistically low co-channel interfernce along the fringes of the simulation area.

Given the above-mentioned 49 cells and that each timeslot my be used either in the UL or DL, the resultant search space size to be explored becomes $2^{49}$, when a new user initiates a call and has to decide about the choice of the serving BS, as well as whether an UL or DL timeslot activation would inflict the lower intereference. As argued before, the size $2^{49}$ of this search-space is excessive, preventing a full search. As a more attractive design option, a GA is utilized for finding a suboptimum, but highly beneficial uplink or downlink TS allocation. The computational complexity of the GA-aided search was set to $P \cdot Y=100$ objective function evaluation, while using different $P$ and $Y$ values. The "No GA" based TDD network was found to support 58 users at $P_{F T}=1 \%$, corresponding to a traffic load of 0.6 Erlang $/ \mathrm{km}^{2} / \mathrm{MHz}$. Upon employing GA-assisted UL/DL timeslot allocation before the transmission of each UTRA frame, the number of users supported by the TDD network increased to 185 users, or to an equivalent traffic load of 2.11 Erlang $/ \mathrm{km}^{2} / \mathrm{MHz}$, when invoking a GA population size of $P=10$ and $Y=10$ generations. When the population size was reduced to $P=4$ in conjunction with $Y=25$ generations, the TDD system was capable of supporting 174 users, corresponding to a teletraffic density of 2.01 Erlang $/ \mathrm{km}^{2} / \mathrm{MHz}$.

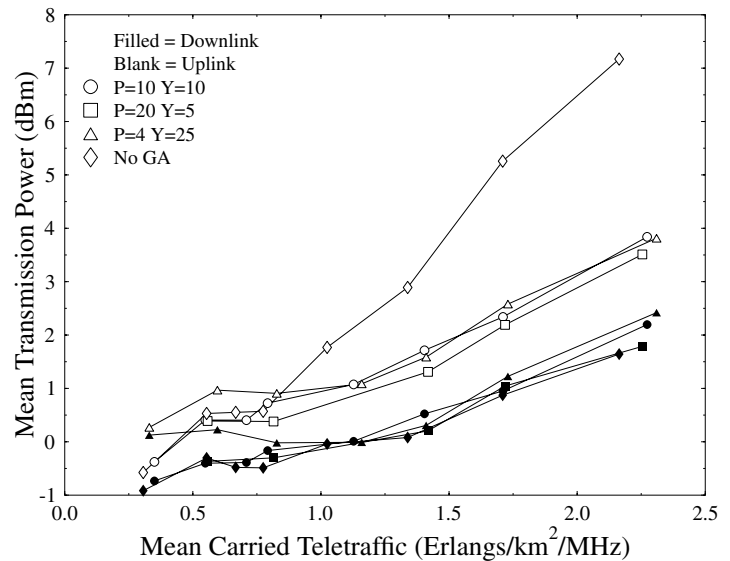

Figure 5: Mean transmission power versus mean carried traffic of the UTRA-like TDD/CDMA based cellular network both with and without GA-assisted UL/DL TS-allocation as well as with shadowing having a standard deviation of $3 \mathrm{~dB}$ for a spreading factor of $S F=16$.

Figure 4 portrays the probability of low quality access versus various traffic loads. It can be seen from the figure that the probability of low quality access for the "No GA" scheme becomes better than that of the systems using GA-assisted UL/DL timeslot scheduling. This is a consequence of the associated high probability of forced termination for the "No GA" scheme, as shown in Figure 3, because the higher the probability of forced termination, the lower the number of users supported by the TDD system. Hence the effects of co-channel interference imposed by the existing connections remain more benign, when a new call is initiated. Hence a better connection quality is maintained compared to that of the "GA-assisted" scheme. From the figure we observe that the GA-aided TDD system's teletraffic density was limited to 1.74 Erlangs $/ \mathrm{km}^{2} / \mathrm{MHz}$, corresponding to 151 users, which was limited by the performance metric $P_{\text {low }}$.

For the sake of characterizing the achievable system performance also from a different perspective, the mean transmission power versus teletraffic performance is depicted in Figure 5. We observe in the figure that both the "GA-assisted" and "No GA" scenario obey a similar trend in terms of their downlink power consumption. However, in terms of uplink power consumption, the "No GA" scheme requires an average of $2 \mathrm{dBm}$ to $5 \mathrm{dBm}$ more signal power than the "GA-assisted" scheme, as the traffic load becomes higher. Again, this is because the severe $B S \rightarrow B S$ inter-cell interference degrades the quality of the call. Hence, for the sake of achieving the target SINR and maintain the existing connections, the MSs have to increase their transmission power, which results in an increased interference level imposed on other connections, hence inflicting a performance degradation upon the whole system. The "GA-assisted" system is capable of avoiding the presence of severe interference by advantageously scheduling the UL/DL timeslots, hence keeping the system's average interference load as low as possible, hence indirectly allowing us to support more MSs.

Figure 6 shows the ratio of uplink to downlink timslots versus various traffic loads. In the context of the "No GA" scheme we fixed the uplink to downlink timeslot utilization ratio to 0.875 , since there are seven uplink timeslots and eight downlink timeslots in each frame. By contrast, in the "GA-assisted" scheme we did not specify the uplink to downlink timeslot ratio. The GA-assisted timeslot scheduling scheme determined, whether a timeslot was used in the uplink or downlink of 


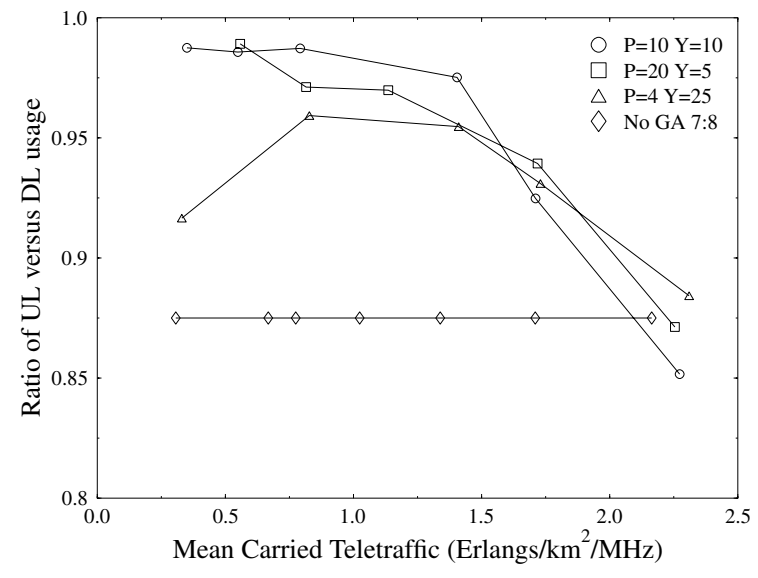

Figure 6: Ratio of uplink timeslots to downlink timeslots versus the mean carried traffic of the UTRA-like TDD/CDMA based cellular network both with and without GA-assisted UL/DL TS allocation and with shadowing having a standard deviation of $3 \mathrm{~dB}$ for a spreading factor of $S F=16$.

the system. From the resultant statistical results we observe that the UL/DL ratio of the "GA-assisted" schemes was between 0.9 and 1.0, which is close to the symmetric traffic load allocation, except for high traffic loads in excess of 2 Erlang $/ \mathrm{km}^{2} / \mathrm{MHz}$.

\section{SUMMARY AND CONCLUSIONS}

In this paper, we introduced a GA-assisted UL/DL timeslot scheduling scheme for the sake of avoiding the severe inter-cell interference caused by using the UTRA TDD/CDMA air interface. The system model and simulation parameters used in this contribution were highlighted in Section 2 and in [1]. The GA aided UTRA TDD/CDMA system's performance was then examined using computer simulations in Section 3. Summaries of the various parameters and the GA configuration that were used in our simulations were listed in Tables 1 and 2 . Significant system performance gains have been achieved by employing the GA-aided UL/DL TS scheduling scheme, as seen in Figure 3. The "No GA" based TDD network was found to support 58 users at $P_{F T}=1 \%$, corresponding to a traffic load of 0.6 Erlang $/ \mathrm{km}^{2} / \mathrm{MHz}$. Upon employing GA-assisted UL/DL timeslot allocation in conjunction with the computational complexity of $P \cdot Y=100$, while using a population size of $P=10$ and $Y=10$ generations, the number of users supported by the TDD network increased to 185 , or to an equivalent traffic load of 2.11 Erlang $/ \mathrm{km}^{2} / \mathrm{MHz}$. We observed in Figure 4 that it was the probability of low quality access, not the probability of forced termination, which imposed the more severe constraint on the system's capacity. In Figure 5, we compared the power consumption of the "No GA" and the "GA-assisted" TDD system. We observed a similar trend in terms of their downlink power consumption. However, in terms of uplink power consumption the "No GA" scheme requires on average approximately $2 \mathrm{dBm}$ to $5 \mathrm{dBm}$ more signal power than the "GA-assisted" scheme, as the traffic load is increased. Our future research is focussed on the asymmetric GAassisted UTRA TDD/CDMA system's achievable performance using both AQAM and beamforming.

\section{REFERENCES}

[1] S. Ni, J. S. Blogh, and L. Hanzo, "On the network performance of UTRA-like TDD and FDD CDMA systems using adaptive modulation and adaptive beamforming," in Proceedings of the IEEE Vehicular Technology Conference VTC 2003 Spring, (Jeju, Korea), pp. 606-610, April, 2003.

[2] L. Hanzo, L. L. Yang, E. L. Kuan, and K. Yen, Single- and Multicarrier DS-CDMA. John Wiley and IEEE Press, 2003.

[3] L. Hanzo, C.H. Wong, and M.S. Yee, Adaptive wireless transceivers: Turbo-Coded, Turbo-Equalised and Space-Time Coded TDMA, CDMA and OFDM systems. John Wiley and IEEE PRESS, 2002.

[4] X. Wu, L.-L. Yang, and L. Hanzo, "Uplink capacity investigations of TDD/CDMA," in Proceedings of the IEEE Vehicular Technology Conference, VTC Spring 2002, vol. 2, (Birmingham, Alabama, USA), pp. 997-1001, 6-9 May 2002.

[5] J. S.Blogh and L. Hanzo, Third-Generation Systems and Intelligent Wireless Networking - Smart Antennas and Adaptive Modulation. John Wiley and IEEE Press, 2002.

[6] D. E. Goldberg, Genetic Algorithms in Search, Optimization, and Machine Learning. ISBN 0201157675, MA USA: AddisonWesley, August 2001.

[7] J. Holland, Adaptation in Natural and Artificial Systems. Ann Arbor, Michigan: University of Michigan Press, 1975.

[8] A. Wolfgang, N. N. Ahmad, S. Chen, and L. Hanzo, "Genetic algorithm assised minimm bit error rate beamforming," in CDROM of the IEEE 59th Vehicular Technology Conference, (Milan, Italy), May, 2004.

[9] M. Alias, A. Samingan, S. Chen, and L. Hanzo, "Multiple antenna aided OFDM employing minimum bit error rate multiuser detection," IEE Electronics Letters, vol. 39, pp. 1769-1770, November 2003.

[10] E. Hou, N. Ansari, and H. Ren, "A Genetic Algorithm for Multiprocessor Scheduling," IEEE Transaction on Parallel and Distributed Systems, vol. 5, pp. 113 - 120, February 1994.

[11] K.-S. Tang, K.-T. Ko, K. F. Man, and S. Kwong, "Topology Design and Bandwidth Allocation of Embedded ATM Networks Using Genetic Algorithms," IEEE Communications Letters, vol. 2, pp. 171 - 173, June 1998.

[12] C. Y. Ngo and V. O. K. Li, "Fixed Channel Assignment in Cellular Radio Network Using a Modified Genetic Algorithm," IEEE Transactions on Vehicular Technology, vol. 47, pp. 163 - 172, Feburary 1998.

[13] D. Sadot, U. Mahlab, and V. Natan-Bar, "New method for developing optical CDMA address code sequences using the genetic algorithm," in Proceedings of the IEEE International Conference on Communication, ICC1998, vol. 3, (Atlanta, GA, USA), pp. 711, June 1998.

[14] Y. H. Lee, S. T. Sheu, and M. H. Chen, "Application of Genetic Algorithm for Optimal Simultaneous Code Set Selection in Optical CDMA," in Modelling and Simulation, MS99, vol. 3, (Philadelphia, USA), pp. 5 - 8, May 1999.

[15] S. H. Hwang and L. Hanzo, "Reverse-link performance of synchronous DS-CDMA systems in dispersive Rician multipath fading channels," IEE Electronics Letters, vol. 39, pp. 1682-1684, November 2003. 